\title{
METABOLIC PROFILE IN PLASMA AND CSF OF LEVODOPA-INDUCED DYSKINESIA OF PARKINSON'S DISEASE
}

Bruno L. Santos-Lobato ${ }^{\mathrm{a}, \mathrm{b}}$, Luiz Gustavo Gardinassi ${ }^{\mathrm{c}}$, Mariza Bortolanza ${ }^{\mathrm{d}}$, Ana Paula Ferranti Peti $^{\mathrm{e}}$, Ângela V. Pimentel ${ }^{\mathrm{a}}$, Lúcia Helena Faccioli ${ }^{\mathrm{e}}$, Elaine A. Del-Bel ${ }^{\mathrm{d}}$, Vitor Tumas ${ }^{\mathrm{a}}$

${ }^{\mathrm{a}}$ Department of Neurosciences and Behavioral Sciences, Ribeirão Preto Medical School, University of São Paulo, Ribeirão Preto, SP, Brazil.

${ }^{\mathrm{b}}$ Laboratório de Neuropatologia Experimental, Federal University of Pará, Belém, PA, Brazil.

${ }^{c}$ Department of Biosciences and Technology, Institute of Tropical Pathology and Public Health, Federal University of Goiás, Goiânia, GO, Brazil.

${ }^{\mathrm{d}}$ Department of Basic and Oral Biology, Faculty of Odontology of Ribeirão Preto, University of São Paulo, Ribeirão Preto, SP, Brazil.

${ }^{\mathrm{e}}$ Department of Clinical Analysis, Toxicology and Food Sciences, School of Pharmaceutical Sciences of Ribeirão Preto, University of São Paulo, Ribeirão Preto, SP, Brazil.

+ Corresponding Authors:

Dr. Elaine Del-Bel

Department of Basic and Oral Biology

Faculty of Odontology of Ribeirão Preto, University of São Paulo

Av do Café, S/N, CEP: 14049-900 - Ribeirão Preto, São Paulo, Brazil

Phone: +55 163315 4050, E-mail: eadelbel@ forp.usp.br

Dr. Vitor Tumas 
Department of Neurosciences and Behavioral Sciences

Ribeirão Preto Medical School, University of São Paulo

Av. Bandeirantes 3900, CEP: 14049-900 - Ribeirão Preto, São Paulo, Brazil

Phone: + 5516 3602-2557 E-mail: tumasv@ rnp.fmrp.usp.br

Word Count: 3255

Running Title: Metabolic profile in dyskinesias

Keywords: Metabolomics, Parkinson's disease, dyskinesia, levodopa

Financial Disclosure: Dr. TUMAS received honoraria from Teva Brasil, UCB Biopharma and Ipsen, and travel support for medical conferences from Roche.

Funding Sources for the Study: This study was supported by the Fundação de Amparo à Pesquisa do Estado de São Paulo (FAPESP; 159688/2015-9 and 2014/07125-6) and Conselho Nacional de Desenvolvimento Científico e Tecnológico (CNPq; 2012/17626-7). The BioFIND study funding was provided by The Michael J. Fox Foundation for Parkinson's Research and National Institute of Neurological Disorders and Stroke. 


\section{Structured Abstract:}

Background: The existence of few biomarkers and the lack of a better understanding of the pathophysiology of levodopa-induced dyskinesia (LID) in Parkinson's disease (PD) require new approaches, as the metabolomic analysis, for discoveries. Objectives: We aimed to identify a metabolic profile associated with LID in patients with PD in an original cohort, and to confirm the results in an external cohort (BioFIND). Methods: In the original cohort, plasma and CSF were collected from 20 healthy controls, 23 patients with PD without LID, and 24 patients with PD with LID. LC-MS/MS and metabolomics data analysis were used to perform untargeted metabolomics. Untargeted metabolomics data from the BioFIND cohort were analyzed. Results: We identified a metabolic profile associated with LID in PD, composed of multiple metabolic pathways. In particular, the dysregulation of glycosphingolipids metabolic pathway was more related to LID and was strongly associated with the severity of dyskinetic movements. Further, bile acid biosynthesis and C21-steroid hormone biosynthesis metabolites simultaneously found in plasma and CSF have distinguished patients with LID from other participants. Levels of cortisol and cortisone were reduced in patients with PD and LID compared to patients with PD without LID. Data from the BioFIND cohort confirmed dysregulation in plasma metabolites from the bile acid biosynthesis and C21-steroid hormone biosynthesis pathways. Conclusion: There is a distinct metabolic profile associated with LID in PD, both in plasma and CSF, which may be associated with the dysregulation of lipid metabolism and neuroinflammation. 


\section{Introduction}

Levodopa-induced dyskinesias (LID) are a major motor complication in Parkinson's disease (PD) after chronic use of levodopa, occurring in 33 to $51.2 \%$ of patients with PD after 5 years of levodopa therapy [1] and causing increased health care costs and worsening of the quality of life [2-5]. Variables as age at PD onset and levodopa dose are important clinical risk factors associated with LID [6,7], as well as the association of some genetic polymorphisms [7]. Maladaptive plastic changes on the basal ganglia neurotransmission [8] and neuroinflammation [9-14] are the main processes associated with LID pathophysiology.

The development of biomarkers in neurological diseases is essential for a better understanding of its pathophysiology, but there are few biomarkers associated with LID in PD. For the discovery of a possible signature and molecular pathogenesis of LID in PD, untargeted metabolomics may be a strategic initial approach concerned with the identification and quantification of metabolites and metabolic pathways linked to a specific condition [15].

Previous studies have mostly explored metabolomics in blood and CSF of patients with PD, and a broad number of metabolic pathway dysfunctions have been associated with the disease [15-18]. Also, PD progression could be estimated based on a panel of metabolites discovered by metabolomic analysis [19], and no difference was seen in the metabolic profile of genetic forms of PD [20]. About metabolomic studies in LID, a previous study showed no association between dyskinesias and the kynurenine pathway in plasma [21], even though another study reported a specific metabolite signature based on kynurenine products in plasma and CSF on LID [22].

To explore metabolites as possible biomarkers of LID, we conducted a study to identify a metabolite profile in plasma and CSF associated with LID in patients with PD. After, we explored the metabolite profile in plasma and CSF associated with LID in another cohort (BioFIND cohort).

\section{Methods}

\section{Study design and participants}


We conducted an observational cross-sectional study to analyze the metabolic profile in plasma and CSF of patients with PD with and without LID and in healthy controls. For more details about the enrollment of participants, inclusion and exclusion criteria, and clinical evaluations, see Marchioni et al. [23]. For evaluation, we collected clinical and epidemiological data, Hoehn and Yahr stage, the MDS-UPDRS, and the Unified Dyskinesia Rating Scale (UDysRS). For analysis, participants were divided into three groups: healthy controls (HC), patients with PD without LID (PD-ND), and patients with PD with LID (PDD). The study was approved by the Ribeirão Preto Medical School Ethics Committee (Number 3.036.243), and all participants provided written informed consent.

Additionally, we used the database from the Fox Investigation for New Discovery of Biomarkers in Parkinson's Disease ("BioFIND”) study cohort (http://biofind.loni.usc.edu/), a cross-sectional and observational study of moderate to advanced patients with PD and healthy controls, conceived for biomarker discovery in PD [24]. For up-to-date information on the study, visit www.michaeljfox.org/biospecimens.

\section{Collection, processing, and storage of biologic samples}

As previously described [23], all patients were instructed to take their regular morning dose of dopaminergic drugs, and we collected peripheral blood by venipuncture and CSF through lumbar puncture on the same day. After, whole blood was centrifuged at $4{ }^{\circ} \mathrm{C}$ and $1600 \times \mathrm{g}$ for 15 minutes; CSF was gently mixed to avoid gradient effects and centrifuged at $4^{\circ} \mathrm{C}$ at $4000 \times \mathrm{g}$ for 10 minutes. Supernatant plasma from whole blood and centrifuged CSF samples were aliquoted into $1-\mathrm{mL}$ cryotubes and stored at $-80^{\circ} \mathrm{C}$ until use. In the BioFIND cohort, blood plasma samples and CSF samples were also collected and immediately frozen in a $-80^{\circ} \mathrm{C}$ freezer [24].

\section{Sample preparation for untargeted metabolomic analysis and metabolite quantification by LC-MS/MS}

Samples were extracted using solid-phase extraction (SPE) as described previously [25] for the extraction of eicosanoids in human plasma. The samples were spiked with $10 \mu \mathrm{L}$ internal 
standards (IS) (corticosterone- $\mathrm{d}_{4}$ at $50 \mathrm{ng} \cdot \mathrm{mL}^{-1}$ in methanol). Following the extraction, the samples were dried, suspended in $50 \mu \mathrm{L}$ of methanol, and injected into the LC-MS/MS system. Before the extraction process, $300 \mu \mathrm{L}$ of plasma samples with IS were denatured with $1.5 \mathrm{~mL}$ of methanol/acetonitrile $(1: 1, \mathrm{v} / \mathrm{v})$ at $4^{\circ} \mathrm{C}$. The denatured proteins were removed by centrifugation $(400 \times \mathrm{g}, 20 \mathrm{~min})$, and the supernatants were diluted in water to decrease the methanol/acetonitrile concentration to $10 \%$. SPE was using C18 SPE cartridges, and the first step consisted of conditioning the cartridge with $2 \mathrm{~mL}$ of methanol and $2 \mathrm{~mL}$ of an aqueous solution of acetic acid $0.1 \%$. After, the samples were loaded, and $2 \mathrm{~mL}$ of an aqueous solution of acetic acid $0.1 \%$ was used for the removal of impurities. For sample elution were used $2 \mathrm{~mL}$ of methanolic solution of acetic acid $0.1 \%$

\section{Metabolomics data analysis}

Untargeted metabolomics data was performed with full scan mass spectral data acquired in positive mode, with mass-to-charge ratio $(\mathrm{m} / \mathrm{z})$ ranging from $100-1500$, as previously described [26]. The package apLCMS [27] was used for peak peaking, noise filtering, retention time and $\mathrm{m} / \mathrm{z}$ alignment, and feature quantification. A metabolite feature is characterized by three parameters: $\mathrm{m} / \mathrm{z}$, retention time, and intensity. Data were $\log 2$ transformed and normalized by the mean. Only features detected in $90 \%$ of plasma (4423 metabolite features) and CSF (4259 metabolite features) were retained for statistical analyses. Missing values were imputed by half mean of a particular feature across all samples. The mummichog software v1.0.10 was used for metabolic pathway enrichment analysis (mass accuracy under $10 \mathrm{ppm})[28]$.

Untargeted metabolomics data from the BioFIND study cohort was acquired and processed by the company Metabolon Inc. Scaled and median normalized data were $\log 2$ transformed and used for statistical analysis.

\section{LC-MS/MS and MRM ${ }^{\mathrm{HR}}$ analysis for quantification of plasma and CSF cortisol and cortisone levels}


To confirm dysregulation of the C21-steroid hormone biosynthesis pathway through two metabolites (cortisol and cortisone), LC-MS/MS analysis was performed according to the methodology described by Peti et al. [29]. In summary, the LC-MS/MS was carried out on a Nexera Ultra-High Performance Liquid Chromatographic (UHPLC) system by Shimadzu (Kyoto, HO, JP) and a TripleTOF® 5600+ mass spectrometry from AB Sciex (Foster, CA, USA) equipped with a Turbo-V IonSpray and Calibrant Delivery System (CDS), operated in positive mode. Mobile phases used for chromatographic separation were formic acid $0.1 \%$ (A) and acetonitrile, containing $0.1 \%$ formic acid in both. The column was an Ascentis Express C18 (100 x $2.1 \mathrm{~mm} ; 2.7 \mu \mathrm{m})$ from Supelco (St. Louis, MO, USA). A flow rate of 0.5 $\mathrm{mL} \cdot \mathrm{min}^{-1}$ and an injection volume of $10 \mu \mathrm{L}$ were used. The gradient condition was described in Appendix S1 (Table S1). The optimized MS parameters were as follows: gas1: 60 psi; gas2: 40 psi; curtain gas: $25 \mathrm{psi}$; ion spray voltage: $5 \mathrm{kV}$; turbo temperature: $550{ }^{\circ} \mathrm{C}$; $\mathrm{m} / \mathrm{z}$ range: 50 a 400; and dwell time: $100 \mathrm{~ms}$.

$\mathrm{MRM}^{\mathrm{HR}}$ transition used for cortisol and cortisone detection were 363.2166>267.1740 and 361.2010> 163.1115, respectively. MultiQuant ${ }^{\mathrm{TM}}$ 3.0.2 software (Foster, CA, USA) was used for data analysis.

\section{Statistical analysis}

To compare two independent groups, we used the Mann-Whitney test; to compare three or more groups, we performed the Kruskal-Wallis test for continuous variables (followed by Dunn's test for multiple comparisons), and the chi-square test for categorical variables. To compare two continuous variables, we performed Spearman's Rho correlation test. Analyses were performed using SPSS for Windows version 23.0 (SPSS Inc., Chicago, USA), and figures were made using GraphPrism for Windows version 5.0 (GraphPad Software Inc., La Jolla, USA). Also, part of these statistical analyses and visualization of untargeted metabolomics data were performed using the software environment for statistical computing and graphics R v3.4.0 and associated packages such as limma (to calculate differential abundance), gplots (to generate heat maps), amap (to perform hierarchical clustering), ggplot2 (to generate Manhattan and Bubble plots). 


\section{Results}

\section{Clinical and epidemiological data}

In the original cohort, we recruited a total of 67 participants who fulfilled the inclusion and exclusion criteria. We did not perform CSF analysis in 4 patients with PD (with LID - 1 patient, without LID - 3 patients) due to technical problems with lumbar puncture. Data are summarized in Appendix S1 (Table S2).

\section{Plasma and CSF metabolite profile associated with PD and LID}

Our untargeted metabolomics analysis detected over 7000 metabolite features, of which 4423 detected in the plasma and 4259 detected in the CSF were used for statistical analysis. In plasma, 249 metabolites (subset 1) distinguished between PD-D and other groups (PD-ND and HC) (Figure 1A), and in CSF, 382 metabolites (subset 2) distinguished between PD-D and other groups (Figure 1B). These subsets of metabolites provided good differentiation between patients with PD and LID and other groups, both in plasma and in CSF (Figure 1C and 1D). Furthermore, 104 metabolites (subset 3) were detected both in plasma and CSF distinguished between PD-D and other groups.

\section{Metabolic pathways associated with PD and LID}

Using the subsets 1 (plasma) and 2 (CSF) of metabolites associated with LID in PD, we detected dysfunctions in multiple metabolic pathways. In plasma, there were dysfunctions in 11 metabolic pathways: glycosphingolipids metabolism, biopterin metabolism, bile acid biosynthesis, omega-3 fatty acid metabolism, butanoate metabolism, vitamin E metabolism, sialic acid metabolism, selenoamino acid metabolism, saturated fatty acid beta-oxidation, lysine metabolism, and fatty acid metabolism, in decrescent order of significance (Figure 1E). In CSF, there were dysfunctions in 7 metabolic pathways: glycosphingolipids metabolism, glycerophospholipids metabolism, dynorphin metabolism, biopterin metabolism, squalene and cholesterol biosynthesis, phosphatidylinositol phosphate metabolism, and pyrimidine metabolism, in decrescent order of significance (Figure 1F). Considering the metabolites associated with LID in PD in both plasma and CSF (subset 3), we detected dysfunctions in 2 
metabolic pathways: bile acid biosynthesis and C21-steroid hormone biosynthesis and metabolism (Figure 2). Metabolic pathways and metabolites associated with LID in PD are described in Appendix S1 (Table S3).

\section{LID severity is associated with specific metabolic pathways}

To evaluate the association between plasma and CSF metabolites with LID severity, we performed linear regression models adjusted by age, gender, disease duration, and LEDD using UDysRS as a continuous variable and marker of LID severity. After, we used the pvalues extracted from linear regression models to identify the main biochemical pathways associated with LID severity using the mummichog software [28]. There was a match in some metabolic pathways associated with LID by hierarchical clustering analysis and linear regression model in plasma (vitamin E metabolism) and CSF (pyrimidine metabolism, glycosphingolipids metabolism, glycerophospholipids metabolism) (Table 1 and Figure $\mathrm{S} 1$ in Appendix S1).

\section{Metabolites from bile acid biosynthesis and C21-steroid hormone biosynthesis pathways are associated with LID in the BioFIND cohort}

We included BioFIND cohort data from 41 patients with PD (with LID $-\mathrm{n}=21$; without LID $-\mathrm{n}=20$ ) for analysis of plasma metabolites, and 44 patients with PD (with LID $-n=22$; without LID $-\mathrm{n}=22$ ) for analysis of CSF metabolites. For both plasma and CSF samples, 772 metabolites were quantified. Relative metabolite abundances (after normalization) were compared between plasma and CSF samples from patients with and without PD, and only 4 metabolites in plasma showed differences between groups: deoxycholate, taurodeoxycholate, $3 \beta-7 \alpha$-dihydroxy-cholestenoate (bile acid biosynthesis pathway), and corticosterone (C21steroid hormone biosynthesis pathway) (Figure 3).

\section{Plasma and CSF cortisol and cortisone levels in patients with PD and LID}


Aiming to support the association of C21-steroid hormone biosynthesis pathway with LID, we compared plasma and CSF levels of cortisol and cortisone between PD-D, PD-ND and HC groups. Patients with PD and LID had lower plasma and CSF cortisol levels than patients with PD without LID (plasma: ANOVA $F_{2,63}=3.41, p=0.03$; PD-D versus PD-ND, Bonferroni mean difference $=565.4, \mathrm{p}<0.05$; CSF $: \mathrm{F}_{2,63}=4.96, \mathrm{p}=0.01$; PD-D versus PDND, Bonferroni mean difference $=15.9, \mathrm{p}<0.05)$ (Figure 4). Also, patients with PD and LID had lower CSF cortisone levels than patients with PD without LID (CSF: $F_{2,63}=9.61, \mathrm{p}=$ 0.0002; PD-D versus PD-ND, Bonferroni mean difference $=0.35, \mathrm{p}<0.05)$ (Figure 4).

Overall, we found a weak positive correlation between plasma and CSF cortisol levels ( $\mathrm{r}=$ $0.4, \mathrm{p}=0.001, \mathrm{n}=63$ ) and a moderate positive correlation between plasma and CSF cortisone levels $(\mathrm{r}=0.53, \mathrm{p}<0.001, \mathrm{n}=63)$. There were no correlations between clinical variables (age at PD onset, weight, disease duration, levodopa therapy duration, LEDD, MDS-UPDRS, Hoehn and Yahr stage, and UDysRS) and plasma and CSF cortisol and cortisone levels.

\section{Discussion}

Our results showed that there are metabolite profiles associated with LID in PD in segregated subsets of metabolites in plasma and CSF. These metabolite profiles comprise multiple metabolic pathways (in plasma: glycosphingolipid metabolism, biopterin metabolism, bile acid biosynthesis, omega-3 fatty acid metabolism, butanoate metabolism, vitamin E metabolism, sialic acid metabolism, selenoamino acid metabolism, saturated fatty acid betaoxidation, lysine metabolism, and fatty acid metabolism; in CSF: glycosphingolipid metabolism, glycerophospholipid metabolism, dynorphin metabolism, biopterin metabolism, squalene and cholesterol biosynthesis, phosphatidylinositol phosphate metabolism, and pyrimidine metabolism). Glycosphingolipid metabolism was the metabolic pathway more dysregulated in patients with PD with LID. And between these metabolic profiles associated with LID, glycosphingolipid metabolism and glycerophospholipid metabolism pathways in CSF were strongly associated with the severity of LID symptoms in patients with PD, together with the vitamin E metabolism in plasma.

In the subset of metabolites found simultaneously in plasma and CSF, there was also a metabolic profile associated with LID: dysregulation in bile acid biosynthesis and C21steroid hormone biosynthesis and metabolism pathways were able to discriminate patients 
with PD with LID from other groups. Also, plasma metabolites belonging to bile acid biosynthesis and C21-steroid hormone biosynthesis pathways were associated with LID in an independent cohort (BioFIND). We measured the levels of two metabolites of the C21steroid hormone biosynthesis pathway (cortisol and cortisone): CSF cortisol and cortisone levels were lower in patients with PD and LID than in patients with PD without LID.

The association of glycosphingolipid and LID in PD has not been widely explored. In a rat model of LID, sphingolipid metabolism and signaling pathways were dysregulated in the striatum [30]. Also, growing evidence shows the role of sphingolipids/glycosphingolipids in neuroinflammation through its interaction with microglia, chemical mediators of inflammation (cytokines, chemokines), and inflammation-associated signaling pathways $[31,32]$.

In humans, there is no clear association between LID and sphingolipids/glycosphingolipids metabolism, despite some evidence showing the sphingolipid metabolic pathway is enriched in the plasma of patients with PD [17]. The activity of important enzymes associated with the metabolism of glycosphingolipids (glucocerebrosidase, alpha-galactosidase A, betagalactosidase, beta-hexosaminidase) is dysregulated in patients with PD [32]. Furthermore, there are many disorders of sphingolipids/glycosphingolipids metabolism identified as lysosomal storage diseases, as Niemann-Pick disease, Gaucher's disease, Farber's disease, and Krabbe's disease [33]. Gaucher's disease, characterized by the absence of betaglucocerebrosidase (GBA) enzyme and accumulation of glucosylceramide and glucosylsphingosine, has been linked with PD in the last decades due to the discovery of mutations in GBAl as the main risk factor for PD [34], and patients with PD and heterozygous $G B A 1$ mutations present earlier disease onset, increased cognitive impairment and more frequent pain [34]. Patients with PD and GBAl mutations may have a higher risk of developing LID [35,36], but there are also inconclusive results [34]. For these patients, ambroxol therapy may increase GBA activity, and a recent clinical trial reported a slight reduction in motor symptoms after six months of ambroxol in patients with PD and GBA1 mutations, but unfortunately motor complications, as LID, were not evaluated [37].

As an antioxidant, vitamin E was explored in PD clinical trials as a possible neuroprotective therapy against the oxidative stress on its pathophysiology, including the well-known DATATOP (Deprenyl and Tocopherol Antioxidative Therapy of Parkinsonism) study; no study showed an effect on preventing or delaying the progression of the disease [38]. 
Notwithstanding the absence of association between vitamin E metabolism pathway and LID, studies on tardive dyskinesia, a complication of chronic neuroleptic therapy which causes abnormal involuntary movements similar to LID, reported reduction of involuntary movements with the supplementation of vitamin E [38].

There is no direct evidence associating a possible role of bile acids on LID in PD. Previous studies showed bile acids (tauroursodeoxycholic acid, ursodeoxycholic acid) may increase the survival of dopaminergic nigral cells after transplantation through anti-apoptotic mechanisms [39] and reverse mitochondrial dysfunction in LRRK2 mutation carriers [40]. As sphingolipids/glycosphingolipids, bile acids are also implicated in neuroinflammation as antiinflammatory agents, reducing astrocyte activation and expression of interferon-1-beta in a mouse model of PD [41], which may have implications for the pathophysiology of LID in PD.

In patients with $\mathrm{PD}$, a metabolomic study in plasma showed the bile acid biosynthesis pathway was dysregulated in patients with PD, together with other lipid metabolic pathways (glycerolphospholipids, fatty acids), and considering the essential role of bile acids on lipid homeostasis [42], the authors suggested that abnormalities in bile acid may cause disturbances in all lipid metabolism [43]. These findings were supported by another recent study, which reported a significant metabolic change in the bile acids pathway, both in a murine model of PD and in patients with PD [20]. Also, another study proposed that abnormalities in bile acids biosynthesis in patients with PD caused by intestinal dysbiosis, which is common in PD, may dysregulate lipid metabolism [44]. Cerebrotendinous xanthomatosis, a rare inborn error of cholesterol and bile acid metabolism associated with parkinsonism, is caused by the deficiency of sterol 27-hydroxylase (CYP27), leading to the absence of chenodeoxycholate, used in secondary bile acid synthesis, and resulting in deposits of cholesterol and cholestanol in tissues, including the brain [45].

The interaction of steroid hormones with dopaminergic neurotransmission stimulated many animal studies using LID models. Estradiol, corticosterone, and dehydroepiandrosterone showed a consistent antidyskinetic effect on parkinsonian animals with chronic levodopa therapy [46-48]. The administration of finasteride, a 5-alpha reductase inhibitor which blocks the conversion of testosterone into dihydrotestosterone, also reduced dyskinetic movement in a LID animal model [49]. Neuroinflammation has been seen as a main mechanism underlying the steroid-mediated reduction of LID [48]. In patients with PD, a double-blind randomized 
clinical trial of low-dose estrogen replacement therapy showed the same frequency of LID in post-menopausal women with PD using estrogen or not using [50]. Regarding the association between the C21-steroid hormone biosynthesis pathway and PD, a metabolomic study showed a decrease in testosterone levels in the serum of patients with PD [18].

Together, most of these metabolic pathways dysregulated in dyskinetic patients with PD in this study are part of the metabolism of lipids (glycosphingolipids, bile acid biosynthesis, C21-steroid hormone biosynthesis). Considering the role of lipids in neuroinflammation [51], the dysregulation of the lipid metabolism in the plasma and CSF of patients with PD and LID support the hypothesis that a chronic pro-inflammatory state in the brain leads to LID.

As a limitation, we did not include a drug-näive group of patients with $\mathrm{PD}$, which would allow us to investigate variations in the metabolic profile of untreated patients.

In conclusion, these findings suggest that there is a distinct metabolic profile associated with LID in PD, both in plasma and CSF, which may be associated with the dysregulation of lipid metabolism and neuroinflammation.

\section{Acknowledgments}

We would like to thank Manuelina Macruz Capelari, Nathália Novaretti, and Larissa Serveli for technical support.

Parts of the data used in the preparation of this article were obtained from the Fox Investigation for New Discovery of Biomarkers ("BioFIND") database (http://biofind.loni.usc.edu/). For up-to-date information on the study, visit www.michaelifox.org/biofind. BioFIND is sponsored by The Michael J. Fox Foundation for Parkinson's Research (MJFF) with support from the National Institute for Neurological Disorders and Stroke (NINDS).

\section{Author's Role}

BLSL: 1A, 1B, 1C, 2A, 2B, 2C, 3A, 3B 
LGG: $1 \mathrm{C}, 2 \mathrm{~A}, 2 \mathrm{~B}, 2 \mathrm{C}, 3 \mathrm{~B}$

MB: 1C, 2B, 2C, 3B

APFP: 1C, 3B

AVP: 1C, 3B

LHF: 1C, 2C, 3B

EADB: 1A, 1B, 2C, 3B

VT: 1A, 1B, 2C, 3B

\section{References}

1. Poewe W, Seppi K, Tanner CM, Halliday GM, Brundin P, Volkmann J, et al. Parkinson disease. Nat Rev Dis Primers. 2017;3: 17013.

2. Chapuis S, Ouchchane L, Metz O, Gerbaud L, Durif F. Impact of the motor complications of Parkinson's disease on the quality of life. Mov Disord. 2005;20: 224230 .

3. Hechtner MC, Vogt T, Zöllner Y, Schröder S, Sauer JB, Binder H, et al. Quality of life in Parkinson's disease patients with motor fluctuations and dyskinesias in five European countries. Parkinsonism Relat Disord. 2014;20: 969-974.

4. Perez-Lloret S, Negre-Pages L, Damier P, Delval A, Derkinderen P, Destée A, et al. LDOPA-induced dyskinesias, motor fluctuations and health-related quality of life: the COPARK survey. Eur J Neurol. 2017;24: 1532-1538.

5. Pechevis M, Clarke CE, Vieregge P, Khoshnood B, Deschaseaux-Voinet C, Berdeaux G, et al. Effects of dyskinesias in Parkinson's disease on quality of life and health-related costs: a prospective European study. European Journal of Neurology. 2005. pp. 956-963. doi:10.1111/j.1468-1331.2005.01096.x

6. Kelly MJ, Lawton MA, Baig F, Ruffmann C, Barber TR, Lo C, et al. Predictors of motor complications in early Parkinson's disease: A prospective cohort study. Mov Disord. 2019;34: 1174-1183.

7. Santos-Lobato BL, Schumacher-Schuh AF, Rieder CRM, Hutz MH, Borges V, Ferraz $\mathrm{HB}$, et al. Diagnostic prediction model for levodopa-induced dyskinesia in Parkinson's disease. Arq Neuropsiquiatr. 2020;78: 206-216. 
8. Calabresi P, Di Filippo M, Ghiglieri V, Tambasco N, Picconi B. Levodopa-induced dyskinesias in patients with Parkinson's disease: filling the bench-to-bedside gap. Lancet Neurol. 2010;9: 1106-1117.

9. Del-Bel E, Bortolanza M, Dos-Santos-Pereira M, Bariotto K, Raisman-Vozari R. 1DOPA-induced dyskinesia in Parkinson's disease: Are neuroinflammation and astrocytes key elements? Synapse. 2016;70: 479-500.

10. Bortolanza M, Cavalcanti-Kiwiatkoski R, Padovan-Neto FE, da-Silva CA, Mitkovski M, Raisman-Vozari R, et al. Glial activation is associated with 1-DOPA induced dyskinesia and blocked by a nitric oxide synthase inhibitor in a rat model of Parkinson's disease. Neurobiol Dis. 2015;73: 377-387.

11. Bortolanza M, Padovan-Neto FE, Cavalcanti-Kiwiatkoski R, Dos Santos-Pereira M, Mitkovski M, Raisman-Vozari R, et al. Are cyclooxygenase-2 and nitric oxide involved in the dyskinesia of Parkinson's disease induced by L-DOPA? Philos Trans R Soc Lond B Biol Sci. 2015;370. doi:10.1098/rstb.2014.0190

12. Mulas G, Espa E, Fenu S, Spiga S, Cossu G, Pillai E, et al. Differential induction of dyskinesia and neuroinflammation by pulsatile versus continuous 1-DOPA delivery in the 6-OHDA model of Parkinson's disease. Exp Neurol. 2016;286: 83-92.

13. Ohlin KE, Francardo V, Lindgren HS, Sillivan SE, O'Sullivan SS, Luksik AS, et al. Vascular endothelial growth factor is upregulated by L-dopa in the parkinsonian brain: implications for the development of dyskinesia. Brain. 2011;134: 2339-2357.

14. Lerner RP, Francardo V, Fujita K, Bimpisidis Z, Jourdain VA, Tang CC, et al. Levodopa-induced abnormal involuntary movements correlate with altered permeability of the blood-brain-barrier in the basal ganglia. Sci Rep. 2017;7: 16005.

15. Stoessel D, Schulte C, dos Santos MCT, Scheller D, Rebollo-Mesa I, Deuschle C, et al. Promising Metabolite Profiles in the Plasma and CSF of Early Clinical Parkinson's Disease. Frontiers in Aging Neuroscience. 2018. doi:10.3389/fnagi.2018.00051

16. Shao Y, Le W. Recent advances and perspectives of metabolomics-based investigations in Parkinson's disease. Mol Neurodegener. 2019;14: 3.

17. Hu L, Dong M-X, Huang Y-L, Lu C-Q, Qian Q, Zhang C-C, et al. Integrated Metabolomics and Proteomics Analysis Reveals Plasma Lipid Metabolic Disturbance in Patients With Parkinson's Disease. Front Mol Neurosci. 2020;13: 80.

18. Toczylowska B, Zieminska E, Michałowska M, Chalimoniuk M, Fiszer U. Changes in the metabolic profiles of the serum and putamen in Parkinson's disease patients - In vitro and in vivo NMR spectroscopy studies. Brain Res. 2020;1748: 147118.

19. LeWitt PA, Li J, Lu M, Guo L, Auinger P, Parkinson Study Group-DATATOP Investigators. Metabolomic biomarkers as strong correlates of Parkinson disease progression. Neurology. 2017;88: 862-869.

20. Yakhine-Diop SMS, Morales-García JA, Niso-Santano M, González-Polo RA, UribeCarretero E, Martinez-Chacon G, et al. Metabolic alterations in plasma from patients with familial and idiopathic Parkinson's disease. Aging . 2020;12: 16690-16708. 
21. Chang K-H, Cheng M-L, Tang H-Y, Huang C-Y, Wu Y-R, Chen C-M. Alternations of Metabolic Profile and Kynurenine Metabolism in the Plasma of Parkinson's Disease. Mol Neurobiol. 2018;55: 6319-6328.

22. Havelund JF, Andersen AD, Binzer M, Blaabjerg M, Heegaard NHH, Stenager E, et al. Changes in kynurenine pathway metabolism in Parkinson patients with L-DOPAinduced dyskinesia. J Neurochem. 2017;142: 756-766.

23. Marchioni C, Santos-Lobato BL, Queiroz MEC, Crippa JAS, Tumas V. Endocannabinoid levels in patients with Parkinson's disease with and without levodopainduced dyskinesias. J Neural Transm. 2020;127: 1359-1367.

24. Kang UJ, Goldman JG, Alcalay RN, Xie T, Tuite P, Henchcliffe C, et al. The BioFIND study: Characteristics of a clinically typical Parkinson's disease biomarker cohort. Mov Disord. 2016;31: 924-932.

25. Galvão AF, Petta T, Flamand N, Bollela VR, Silva CL, Jarduli LR, et al. Plasma eicosanoid profiles determined by high-performance liquid chromatography coupled with tandem mass spectrometry in stimulated peripheral blood from healthy individuals and sickle cell anemia patients in treatment. Anal Bioanal Chem. 2016;408: 3613-3623.

26. Pereira PAT, Bitencourt CS, Reis MB, Frantz FG, Sorgi CA, Souza COS, et al. Immunomodulatory activity of hyaluronidase is associated with metabolic adaptations during acute inflammation. Inflamm Res. 2020;69: 105-113.

27. Yu T, Park Y, Johnson JM, Jones DP. apLCMS--adaptive processing of high-resolution LC/MS data. Bioinformatics. 2009;25: 1930-1936.

28. Li S, Park Y, Duraisingham S, Strobel FH, Khan N, Soltow QA, et al. Predicting network activity from high throughput metabolomics. PLoS Comput Biol. 2013;9: e1003123.

29. Peti APF, Locachevic GA, Prado MKB, de Moraes LAB, Faccioli LH. High-resolution multiple reaction monitoring method for quantification of steroidal hormones in plasma. J Mass Spectrom. 2018;53: 423-431.

30. Wang Y, Zhang G-J, Sun Y-N, Yao L, Wang H-S, Du C-X, et al. Identification of metabolite biomarkers for L-DOPA-induced dyskinesia in a rat model of Parkinson's disease by metabolomic technology. Behavioural Brain Research. 2018. pp. 175-183. doi:10.1016/j.bbr.2018.03.020

31. Gualtierotti R, Guarnaccia L, Beretta M, Navone SE, Campanella R, Riboni L, et al. Modulation of Neuroinflammation in the Central Nervous System: Role of Chemokines and Sphingolipids. Adv Ther. 2017;34: 396-420.

32. Belarbi K, Cuvelier E, Bonte M-A, Desplanque M, Gressier B, Devos D, et al. Glycosphingolipids and neuroinflammation in Parkinson's disease. Mol Neurodegener. 2020;15: 59.

33. Alessenko AV, Albi E. Exploring Sphingolipid Implications in Neurodegeneration. Frontiers in Neurology. 2020. doi:10.3389/fneur.2020.00437

34. Kim H-J, Mason S, Foltynie T, Winder-Rhodes S, Barker RA, Williams-Gray CH. 
Motor complications in Parkinson's disease: 13-year follow-up of the CamPaIGN cohort. Mov Disord. 2020;35: 185-190.

35. Oeda T, Umemura A, Mori Y, Tomita S, Kohsaka M, Park K, et al. Impact of glucocerebrosidase mutations on motor and nonmotor complications in Parkinson's disease. Neurobiol Aging. 2015;36: 3306-3313.

36. Olszewska DA, McCarthy A, Soto-Beasley AI, Walton RL, Magennis B, McLaughlin RL, et al. Association Between Glucocerebrosidase Mutations and Parkinson's Disease in Ireland. Front Neurol. 2020;11: 527.

37. Mullin S, Smith L, Lee K, D'Souza G, Woodgate P, Elflein J, et al. Ambroxol for the Treatment of Patients With Parkinson Disease With and Without Glucocerebrosidase Gene Mutations: A Nonrandomized, Noncontrolled Trial. JAMA Neurol. 2020;77: 427434.

38. Pham DQ, Plakogiannis R. Vitamin E supplementation in Alzheimer's disease, Parkinson's disease, tardive dyskinesia, and cataract: Part 2. Ann Pharmacother. 2005;39: 2065-2072.

39. Duan W-M, Rodrigues CMP, Zhao L-R, Steer CJ, Low WC. Tauroursodeoxycholic acid improves the survival and function of nigral transplants in a rat model of Parkinson's disease. Cell Transplant. 2002;11: 195-205.

40. Mortiboys H, Furmston R, Bronstad G, Aasly J, Elliott C, Bandmann O. UDCA exerts beneficial effect on mitochondrial dysfunction in LRRK2(G2019S) carriers and in vivo. Neurology. 2015;85: 846-852.

41. Mendes MO, Rosa AI, Carvalho AN, Nunes MJ, Dionísio P, Rodrigues E, et al. Neurotoxic effects of MPTP on mouse cerebral cortex: Modulation of neuroinflammation as a neuroprotective strategy. Mol Cell Neurosci. 2019;96: 1-9.

42. Kumari A, Pal Pathak D, Asthana S. Bile acids mediated potential functional interaction between FXR and FATP5 in the regulation of Lipid Metabolism. Int J Biol Sci. 2020;16: 2308-2322.

43. Zhao H, Wang $\mathrm{C}$, Zhao N, Li W, Yang Z, Liu X, et al. Potential biomarkers of Parkinson's disease revealed by plasma metabolic profiling. J Chromatogr B Analyt Technol Biomed Life Sci. 2018;1081-1082: 101-108.

44. Hasuike Y, Endo T, Koroyasu M, Matsui M, Mori C, Yamadera M, et al. Bile acid abnormality induced by intestinal dysbiosis might explain lipid metabolism in Parkinson's disease. Med Hypotheses. 2020;134: 109436.

45. Stelten BML, van de Warrenburg BPC, Wevers RA, Verrips A. Movement disorders in cerebrotendinous xanthomatosis. Parkinsonism Relat Disord. 2019;58: 12-16.

46. Gomez-Mancilla B, Bédard PJ. Effect of estrogen and progesterone on L-dopa induced dyskinesia in MPTP-treated monkeys. Neurosci Lett. 1992;135: 129-132.

47. Bélanger N, Grégoire L, Bédard PJ, Di Paolo T. DHEA improves symptomatic treatment of moderately and severely impaired MPTP monkeys. Neurobiol Aging. 2006;27: 16841693. 
48. Barnum CJ, Eskow KL, Dupre K, Blandino P Jr, Deak T, Bishop C. Exogenous corticosterone reduces L-DOPA-induced dyskinesia in the hemi-parkinsonian rat: role for interleukin-1beta. Neuroscience. 2008;156: 30-41.

49. Frau R, Savoia P, Fanni S, Fiorentini C, Fidalgo C, Tronci E, et al. The 5-alpha reductase inhibitor finasteride reduces dyskinesia in a rat model of Parkinson's disease. Exp Neurol. 2017;291: 1-7.

50. Parkinson Study Group POETRY Investigators. A randomized pilot trial of estrogen replacement therapy in post-menopausal women with Parkinson's disease. Parkinsonism Relat Disord. 2011;17: 757-760.

51. Xicoy H, Wieringa B, Martens GJM. The Role of Lipids in Parkinson's Disease. Cells. 2019;8. doi:10.3390/cells8010027 
Table 1 Association between metabolic pathways and severity of LID in plasma and CSF.

\begin{tabular}{lcccc}
\hline \multicolumn{1}{c}{ Metabolic pathway } & \multicolumn{2}{c}{ Plasma } & \multicolumn{2}{c}{ CSF } \\
\cline { 2 - 5 } & Overlap size & p-value & Overlap size & p-value \\
\hline Glycerophospholipid metabolism & & & 7 & 0.0006 \\
Glycosphingolipid metabolism & 19 & 0.0007 & 7 & 0.0006 \\
Vitamin E metabolism & 13 & 0.0008 & & \\
Linoleate metabolism & & & 4 & 0.001 \\
Selenoamino acid metabolism & 16 & 0.002 & 4 & 0.02 \\
Carnitine shuttle & 8 & 0.002 & 4 & 0.0007 \\
Histidine metabolism & & & 10 & 0.002 \\
Xenobiotics metabolism & & & 3 & 0.003 \\
Glycosphingolipid biosynthesis - Ganglioseries & 13 & 0.004 & 5 & 0.001 \\
Fatty acid activation & 15 & 0.005 & 8 & 0.0005 \\
De novo fatty acid biosynthesis & & & 3 & 0.005 \\
Saturated fatty acids beta-oxidation & 14 & 0.01 & & \\
Methionine and cysteine metabolism & & & 4 & 0.009 \\
Pyrimidine metabolism & & & 3 & 0.009 \\
Drug metabolism - Other enzymes & & & 3 & 0.01 \\
Fructose and mannose metabolism & & & 3 & 0.01 \\
Sialic acid metabolism & & & 6 & 0.01 \\
Leukotriene metabolism & & & 3 & 0.01 \\
Fatty acid metabolism & & & & \\
Parathio degradation & & & & \\
Ascorbate (Vitamin C) and aldarate metabolism & 7 & 0.02 & & 0.0006 \\
Glycine, serine, alanine and threonine & & & 7 & \\
metabolism & & & & \\
\hline
\end{tabular}

Overlap size means the number of metabolites associated with the severity of LID in the metabolic pathway. The p-value represent the significance between the metabolic pathway and the severity of LID. Only pathways with three or more overlapping features were shown. 
medRxiv preprint doi: https://doi.org/10.1101/2020.11.17.20233551; this version posted November 18, 2020. The copyright holder for this preprint (which was not certified by peer review) is the author/funder, who has granted medRxiv a license to display the preprint in perpetuity.

It is made available under a CC-BY-NC 4.0 International license .

Supplement Table 1 Chromatographic gradient used for LC-MS/MS analysis.

Time (min) Mobile Phase A (\%) Mobile Phase B (\%)

\begin{tabular}{lll}
\hline 0 & 70 & 30 \\
\hline 1 & 70 & 30 \\
\hline 2 & 50 & 50 \\
\hline 5 & 40 & 60 \\
\hline 8 & 40 & 60 \\
\hline 9 & 02 & 98 \\
\hline 11 & & 98 \\
\hline
\end{tabular}


Supplement Table 2 Clinical and epidemiological data of patients with Parkinson's disease and healthy controls.

\begin{tabular}{|c|c|c|c|c|}
\hline General characteristics & HC $(n=20)$ & PD-ND (n=20) & PD-D $(n=23)$ & p-value \\
\hline Male sex, \% (n) & $30(6)$ & $75(15)$ & $65.2(15)$ & $0.002^{\mathrm{a}}$ \\
\hline Age at the time of evaluation ${ }^{\mathrm{b}}$ & $63(61-67)$ & $62(53-66)$ & $59(53-64)$ & $0.06^{\mathrm{c}}$ \\
\hline Weight (kg) & NA & $72.5(66-79)$ & $73(60-83)$ & $0.63^{\mathrm{d}}$ \\
\hline Age at onset of $\mathrm{PD}^{\mathrm{b}}$ & NA & $54.5(48-61)$ & $47(42-51)$ & $0.03^{\mathrm{d}}$ \\
\hline Disease duration (years) ${ }^{\mathrm{b}}$ & NA & $8(5-12)$ & $11(8-16)$ & $0.01^{\mathrm{d}}$ \\
\hline Levodopa therapy duration (years) ${ }^{\mathrm{b}}$ & NA & $5(3-8)$ & $8(6-12)$ & $0.05^{\mathrm{d}}$ \\
\hline Levodopa dose at evaluation (mg/day) ${ }^{\mathrm{b}}$ & NA & $500(400-850)$ & $850(750-1200)$ & $0.003^{\mathrm{d}}$ \\
\hline Levodopa equivalent daily dose (mg/day) ${ }^{\mathrm{b}}$ & NA & $950(500-1275)$ & $1400(1090-2050)$ & $0.002^{\mathrm{d}}$ \\
\hline Tremor as initial motor symptom, \% (n) & NA & $70(14)$ & $43.5(10)$ & $0.12^{\mathrm{a}}$ \\
\hline $\begin{array}{l}\text { Tremor dominant phenotype at evaluation, } \\
\% \text { (n) }\end{array}$ & NA & $80(16)$ & $26.1(6)$ & $0.002^{\mathrm{a}}$ \\
\hline MDS-UPDRS Part I ${ }^{\mathrm{b}}$ & NA & $10.5(3-17)$ & $10(8-17)$ & $0.28^{\mathrm{d}}$ \\
\hline MDS-UPDRS Part II ${ }^{\mathrm{b}}$ & NA & $15(5-18)$ & $19(12-25)$ & $0.02^{\mathrm{d}}$ \\
\hline MDS-UPDRS Part III ${ }^{\mathrm{b}}$ & NA & $30.5(25-40)$ & $25(19-34)$ & $0.3^{\mathrm{d}}$ \\
\hline MDS-UPDRS Part IV & NA & $0(0-5)$ & $11(8-15)$ & $<0.001^{\mathrm{d}}$ \\
\hline MDS-UPDRS Total score ${ }^{\mathrm{b}}$ & NA & $57.5(45-73)$ & $64(57-88)$ & $0.04^{\mathrm{d}}$ \\
\hline Hoehn \& Yahr stage ${ }^{\mathrm{b}}$ & NA & $2(2-2)$ & $2(2-3)$ & $0.37^{\mathrm{d}}$ \\
\hline UDysRS Part $1 \mathrm{~B}^{\mathrm{b}}$ & NA & NA & $21(16-25)$ & - \\
\hline UDysRS Part $3^{\mathrm{b}}$ & NA & NA & $14(11-19)$ & - \\
\hline UDysRS Part $4^{\mathrm{b}}$ & NA & NA & $7(4-9)$ & - \\
\hline UDysRS Historical subscore ${ }^{\mathrm{b}}$ & NA & NA & $28(17-39)$ & - \\
\hline UDysRS Objective subscore $^{\mathrm{b}}$ & NA & NA & $20(15-27)$ & - \\
\hline UDysRS Total score ${ }^{\mathrm{b}}$ & NA & NA & $53(36-62)$ & - \\
\hline
\end{tabular}

${ }^{\mathrm{a}}$ Chi-square test comparing frequencies between healthy controls, patients with PD with and without levodopa-induced dyskinesias

${ }^{\mathrm{b}}$ Values in median (interquartile range)

${ }^{\mathrm{c}}$ Kruskal-Wallis test comparing medians between healthy controls, patients with PD with and without levodopa-induced dyskinesias

${ }^{\mathrm{d}}$ Mann-Whitney test comparing medians between patients with and without levodopa-induced dyskinesias

Abbreviations: HC, healthy controls; NA, not applied; PD-D, Patients with PD with levodopa-induced dyskinesias; PD-ND, Patients with PD without levodopa-induced dyskinesias. 
medRxiv preprint doi: https://doi.org/10.1101/2020.11.17.20233551; this version posted November 18, 2020. The copyright holder for this preprint (which was not certified by peer review) is the author/funder, who has granted medRxiv a license to display the preprint in perpetuity.

It is made available under a CC-BY-NC 4.0 International license .

Supplement Table 3 Metabolic pathways and metabolites associated with LID in PD, in plasma and CSF.

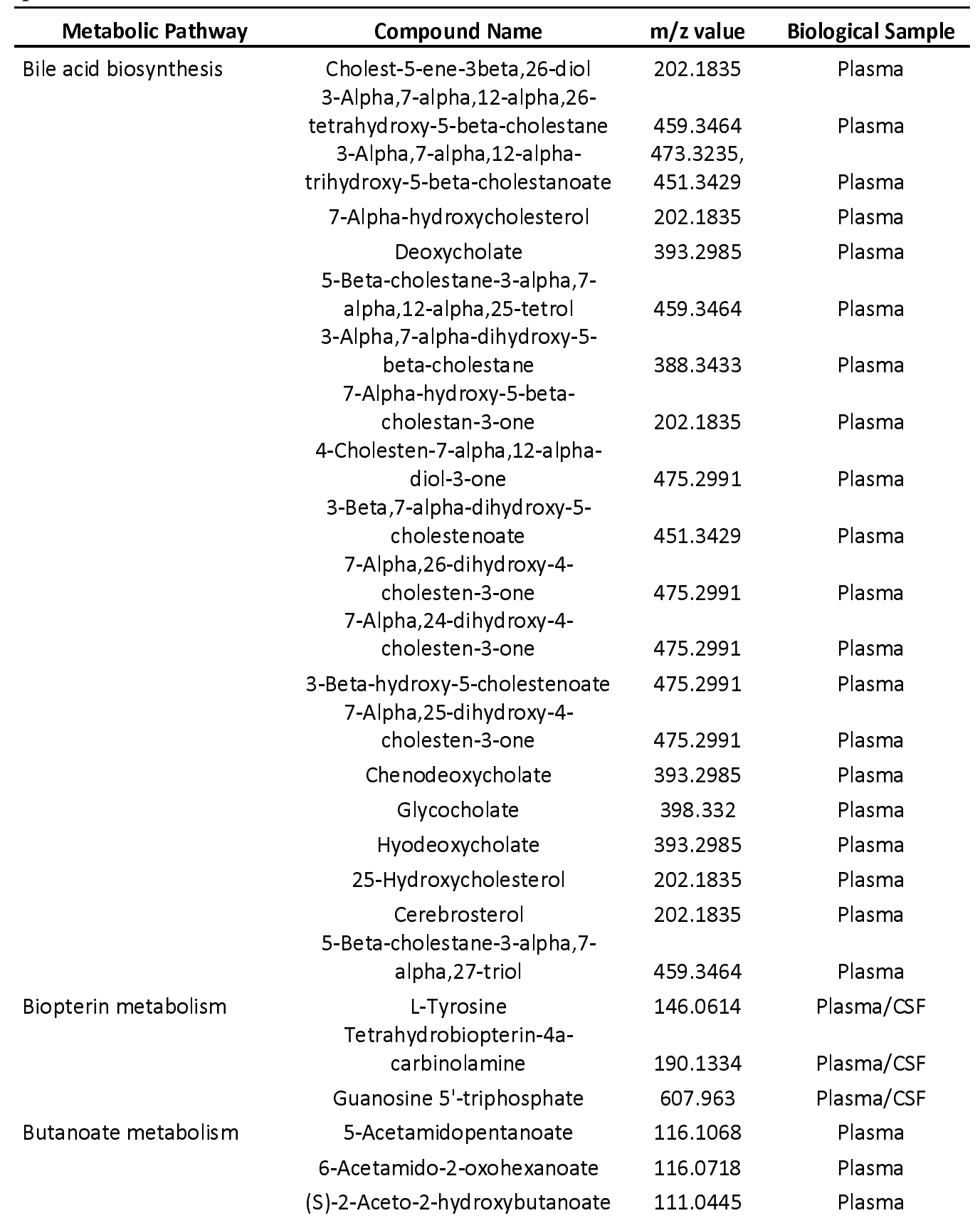


medRxiv preprint doi: https://doi.org/10.1101/2020.11.17.20233551; this version posted November 18, 2020. The copyright holder for this preprint (which was not certified by peer review) is the author/funder, who has granted medRxiv a license to display the preprint in perpetuity.

It is made available under a CC-BY-NC 4.0 International license .

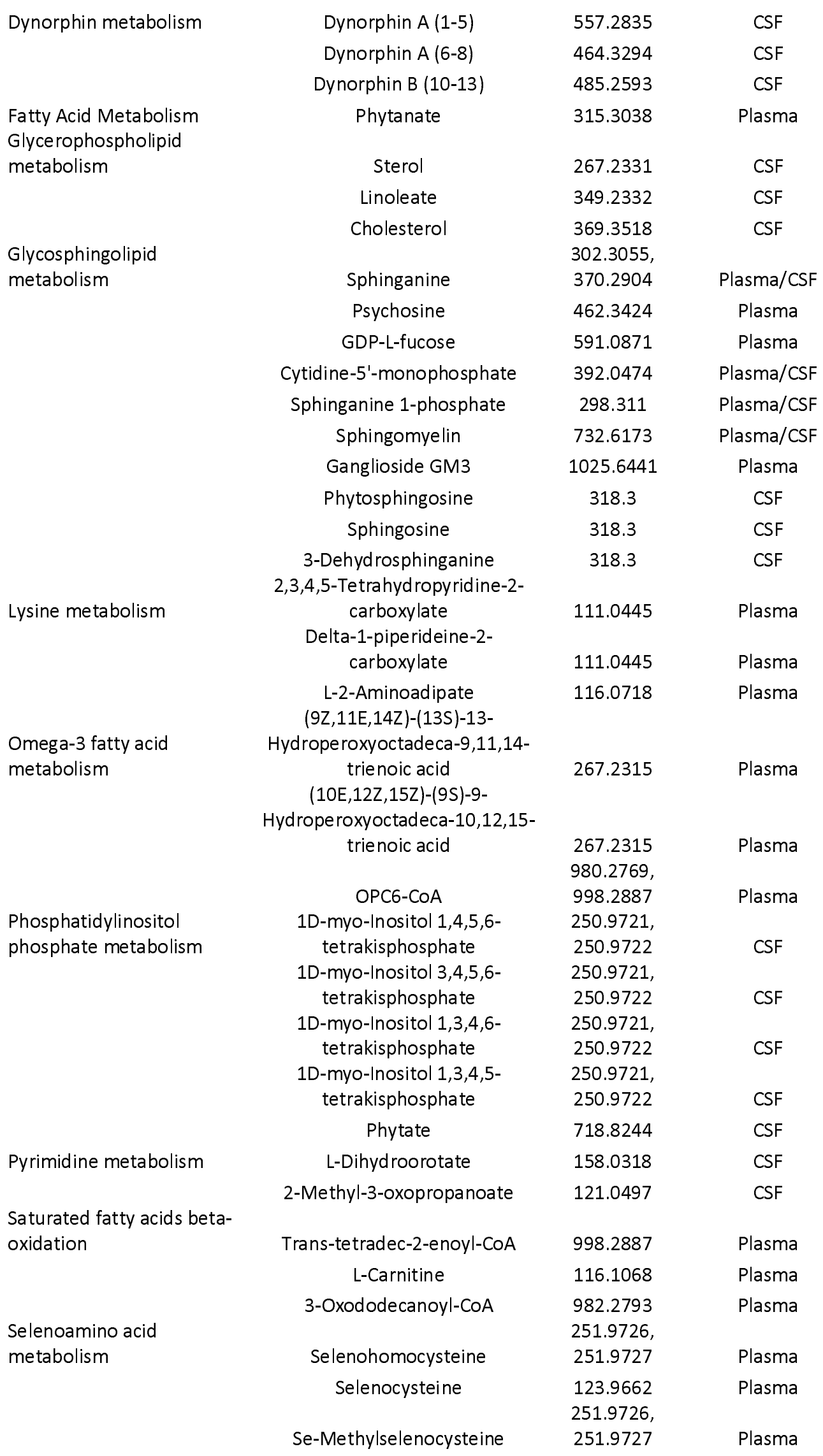


medRxiv preprint doi: https://doi.org/10.1101/2020.11.17.20233551; this version posted November 18, 2020. The copyright holder for this preprint (which was not certified by peer review) is the author/funder, who has granted medRxiv a license to display the preprint in perpetuity.

It is made available under a CC-BY-NC 4.0 International license .

Sialic acid metabolism Squalene and cholesterol biosynthesis

Vitamin E metabolism
D-Galactosyl-3-(N-acetyl-beta-Dgalactosaminyl)-L-serine 453.1679

Plasma

Acetoacetate
7-Dehydrodesmosterol
Campesterol

\section{Presqualene diphosphate} 24,25-Dihydrolanosterol Squalene Lathosterol

Cholesta-5,7,24-trien-3-beta-ol

Cholesta-8(9)-en-3-beta-ol Lanosterol Squalene 2,3-epoxide 5-Dehydroepisterol Cholestenol Alpha-tocopheryl quinone 8-Alpha-hydroxytocopherone 13'-Carboxy-alpha-tocopherol 13'-Hydroxy-alpha-tocopherol 13'-Hydroxy-gama-tocotrienol 13'-Hydroxy-alpha-tocotrienol

\begin{abstract}
1.210 .497
\end{abstract}
4.212 .871

4.593 .356

503.3687,

671.2807

383.403

383.403

3.693 .518

4.212.871

3.693 .518

383.403

383.403

3.693 .518

3.693 .518

485.3435

485.3435

460.3566,

461.362

485.3435

495.31

459.3464
CSF

CSF

CSF

CSF

CSF

CSF

CSF

CSF

CSF

CSF

CSF

CSF

CSF

Plasma

Plasma

Plasma

Plasma

Plasma

Plasma 
medRxiv preprint doi: https://doi.org/10.1101/2020.11.17.20233551; this version posted November 18, 2020. The copyright holder for this preprint (which was not certified by peer review) is the author/funder, who has granted medRxiv a license to display the preprint in perpetuity.

It is made available under a CC-BY-NC 4.0 International license .

Figure 1. Metabolomic analysis of patients with PD with LID. 
medRxiv preprint doi: https://doi.org/10.1101/2020.11.17.20233551; this version posted November 18, 2020. The copyright holder for this preprint (which was not certified by peer review) is the author/funder, who has granted medRxiv a license to display the preprint in perpetuity.

It is made available under a CC-BY-NC 4.0 International license .
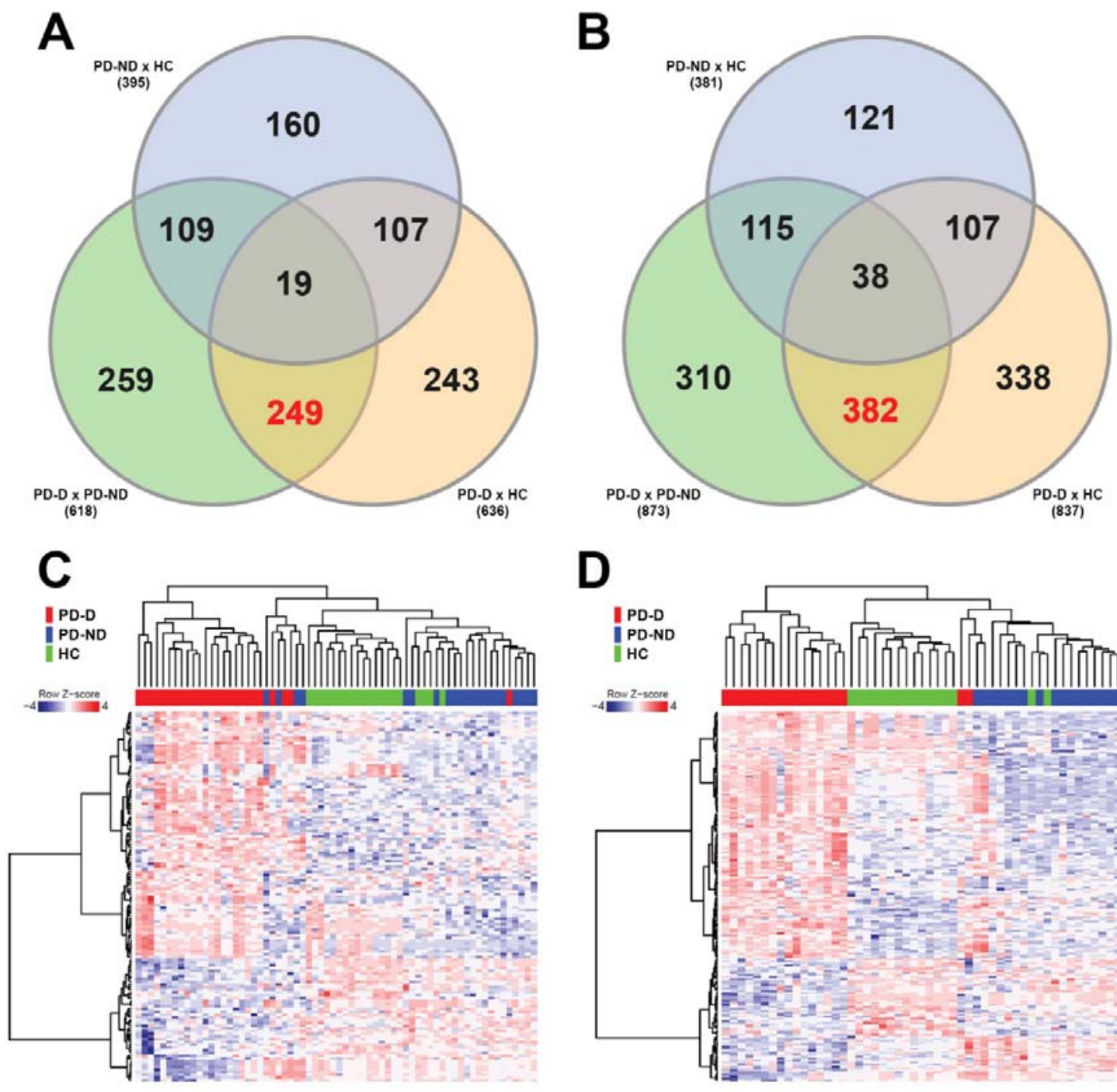

D
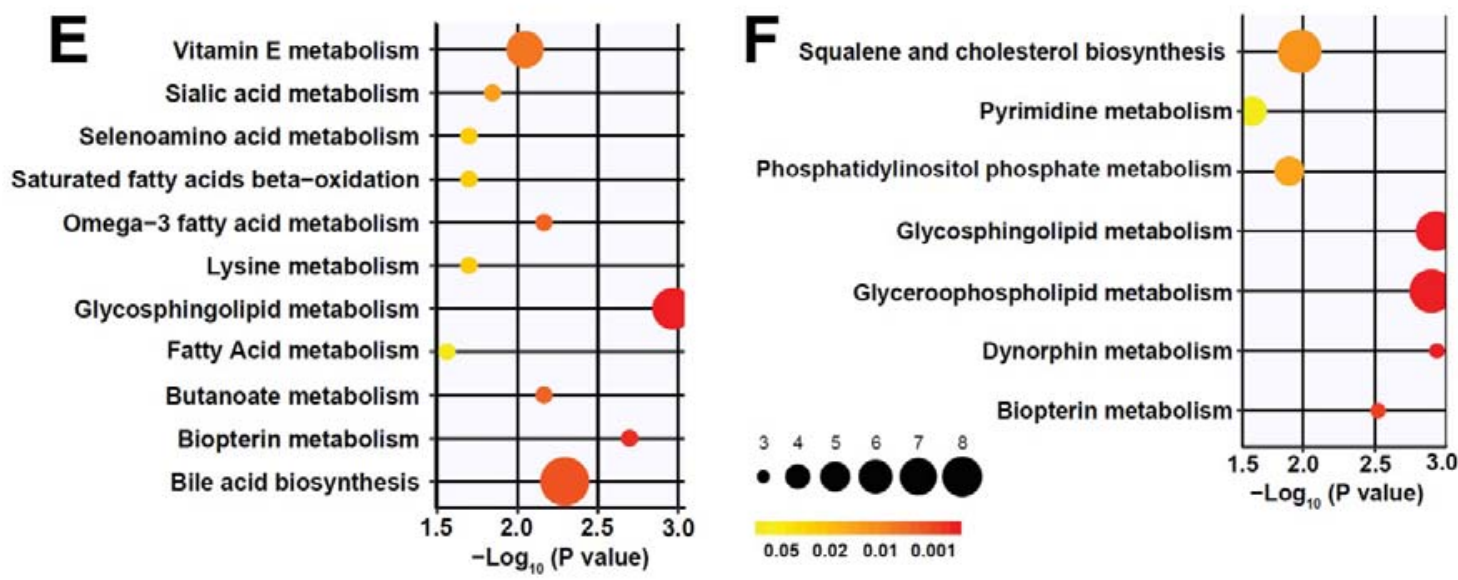

\section{PLASMA}

\section{CSF}


A and B: Venn diagrams of differentially abundant metabolite features between patients with PD and LID (PD-D), patients with PD without LID (PD-ND) and healthy controls (HC), detected in plasma (A) and CSF (B) datasets. Significant features were selected by the moderated t-test with limma package for R. Red values indicate the number of significant metabolites overlapping in comparisons between PD-D vs PD-ND and PD-D vs HC, but not between PD-D vs HC. C and D: Heat maps showing two-way hierarchical clustering based on the intensity of highly significant metabolite features differing between groups in (C) plasma $(135 \mathrm{~m} / \mathrm{z}$ features, $\mathrm{p}<0.001$, FDR $<0.035)$ and (D) CSF $(186 \mathrm{~m} / \mathrm{z}$ features, $\mathrm{p}<0.001$, FDR $<0.025)$. Significant features were selected by ANOVA with limma package for R. The blue-to-red scale indicates lower to higher intensity levels based on a Z-score of normalized intensities for each metabolite feature. E and F: Metabolic pathway analysis showing enriched metabolic pathways in plasma (E) and CSF (F). The color of the circles indicates the significance of the pathway, and the size of the circles indicate the number of metabolites involved in the pathway. 
Figure 2. Metabolic pathway analysis showing enriched pathways in patients with PD and LID in metabolites found both in plasma and CSF.

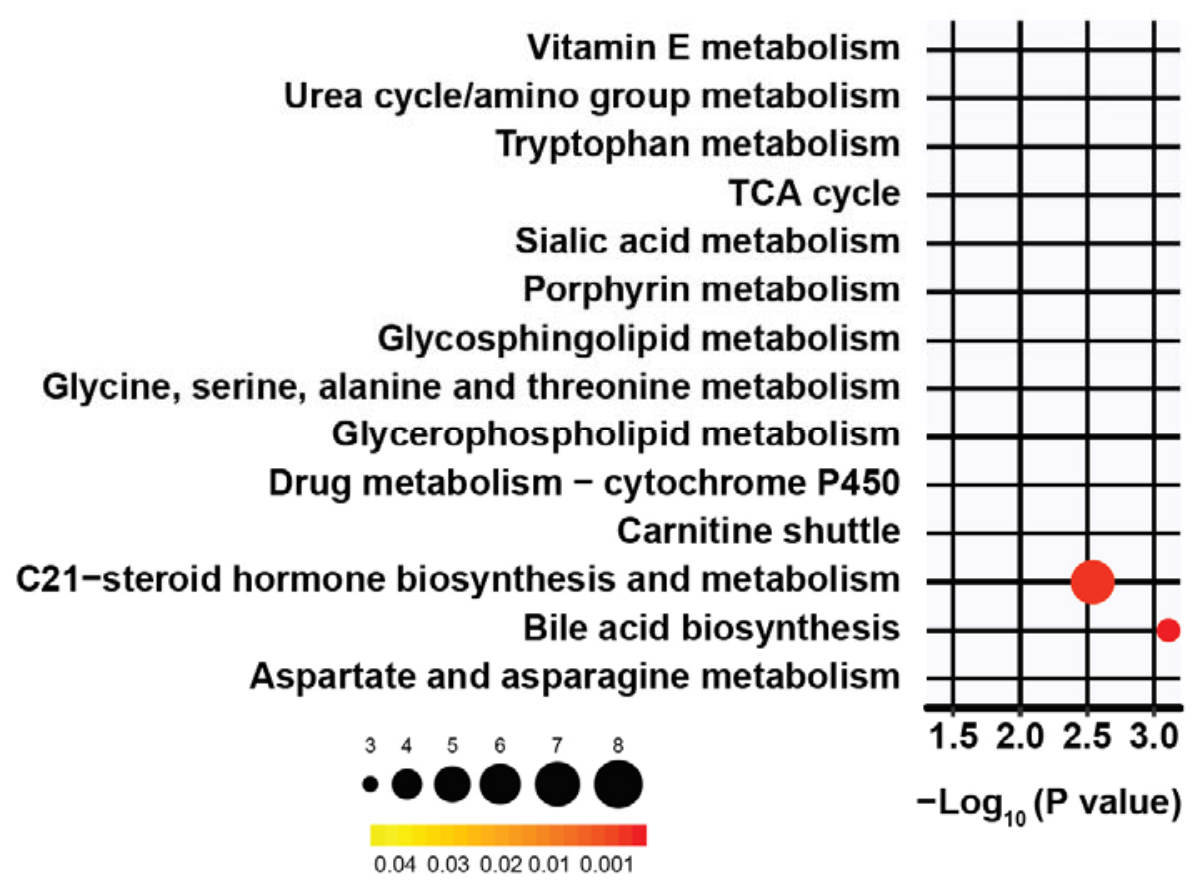

The color of the circles indicates significance of the pathway, and the size of the circles indicate the number of metabolites involved in the pathway. 
Figure 3. Relative abundance of top four metabolites in plasma from participants of BioFIND cohort, according to patients with PD with and without LID.
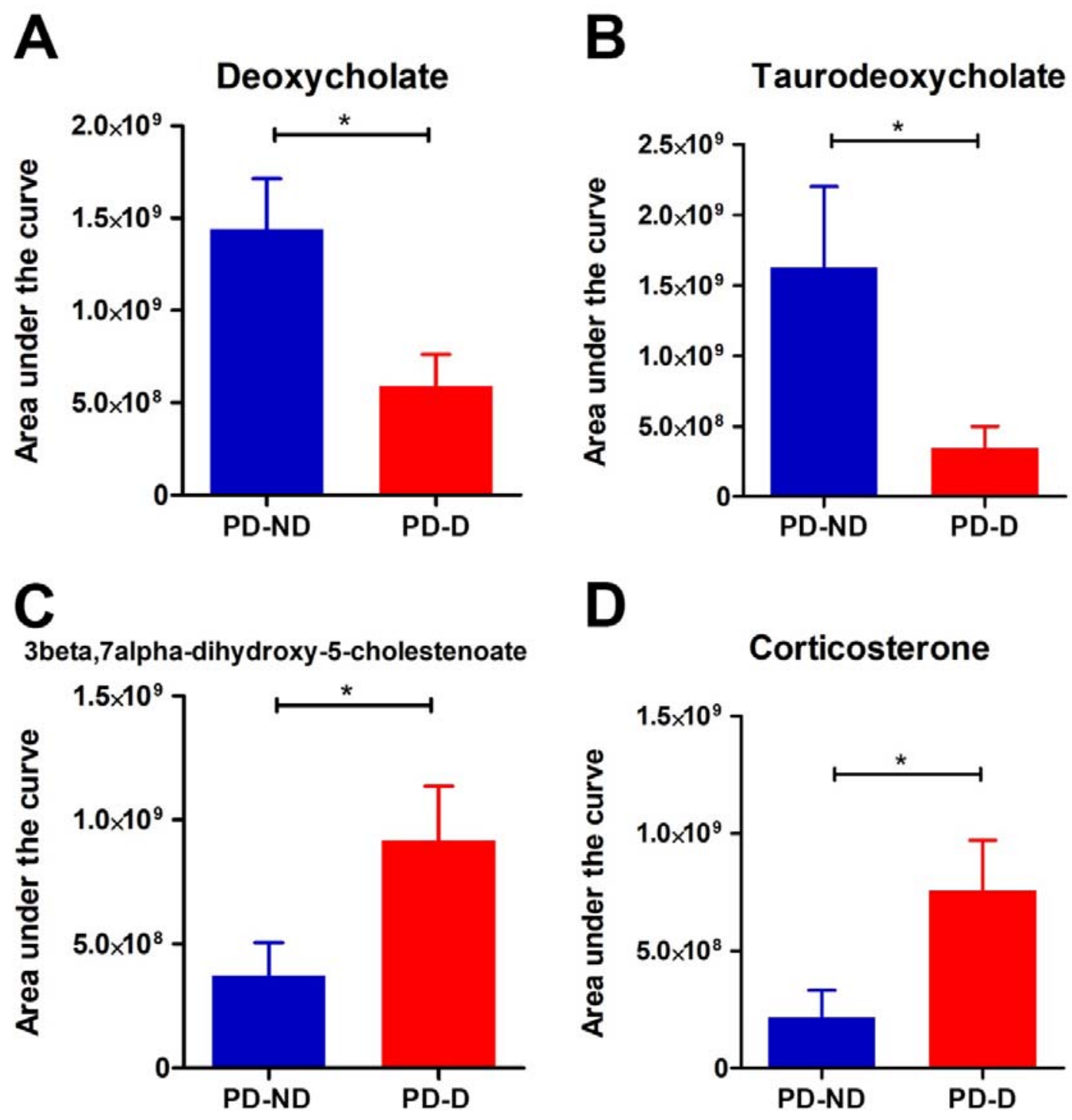

D

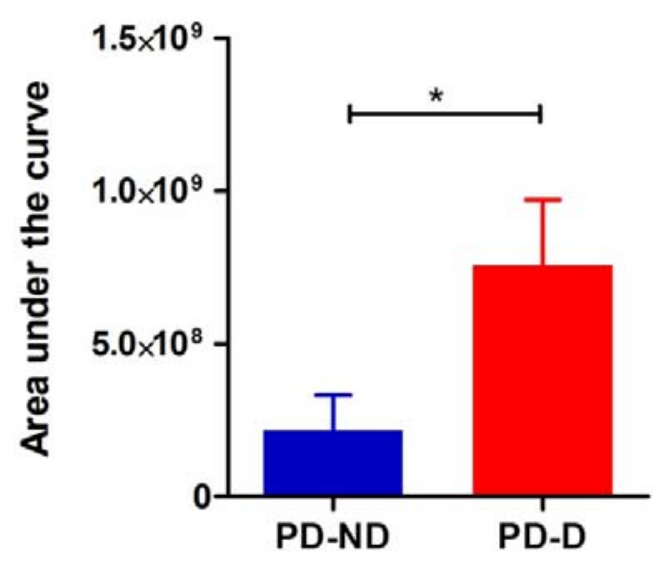

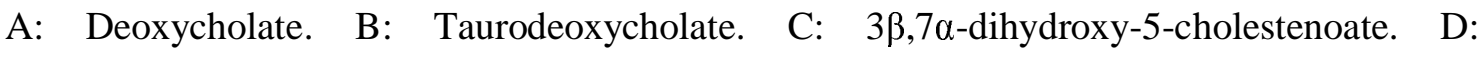
Corticosterone. 
Figure 4. Plasma and CSF cortisol and cortisone levels in patients with PD and healthy controls.
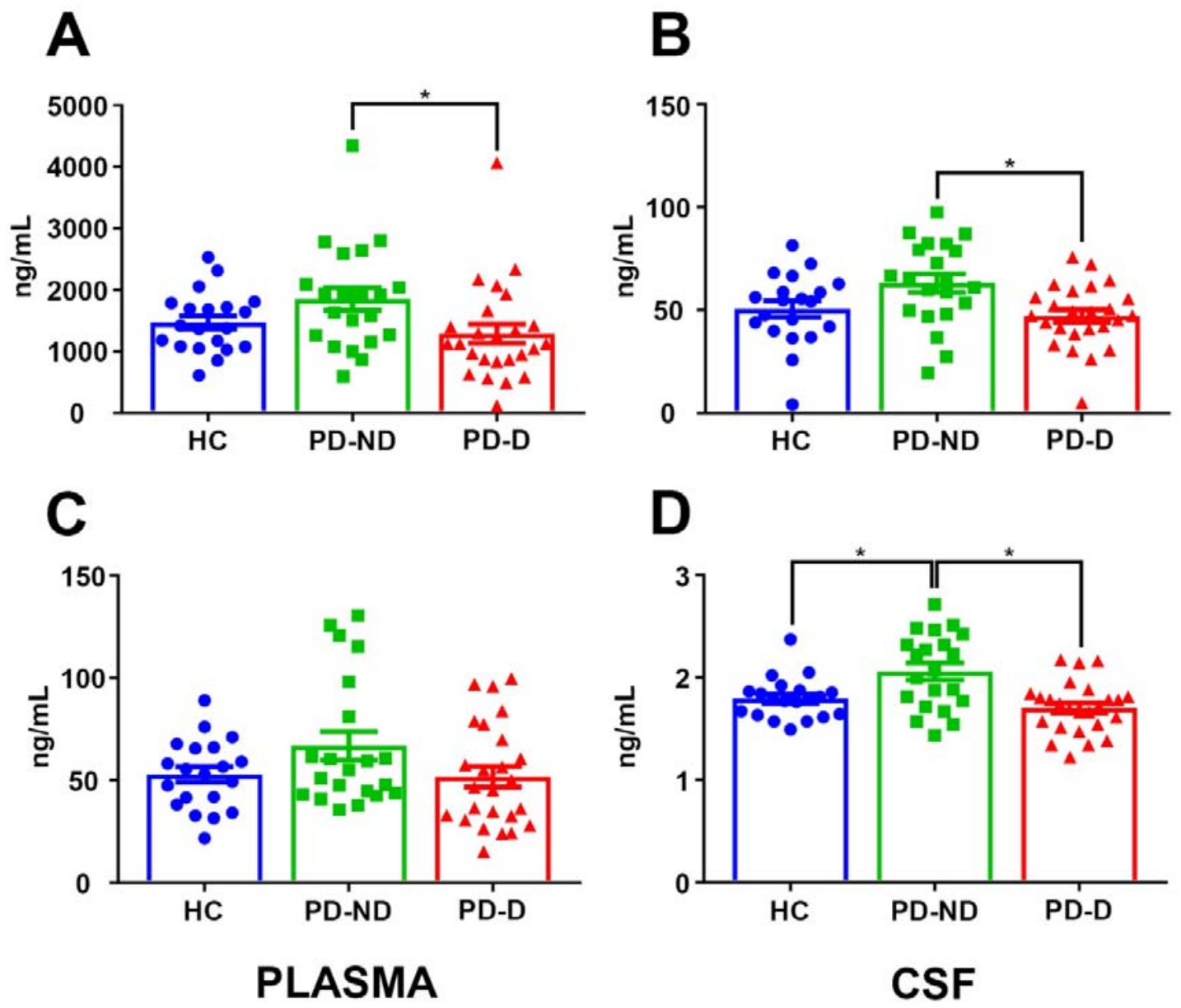

A and B: Cortisol. C and D: Cortisone. 
Supplementary Figure 1. Correlations between p-values of the association of metabolic pathways with LID presence and the association of metabolic pathways with LID severity.

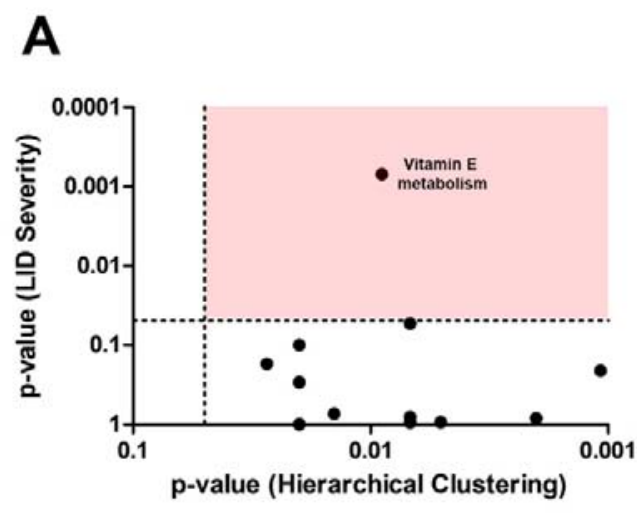

PLASMA
B

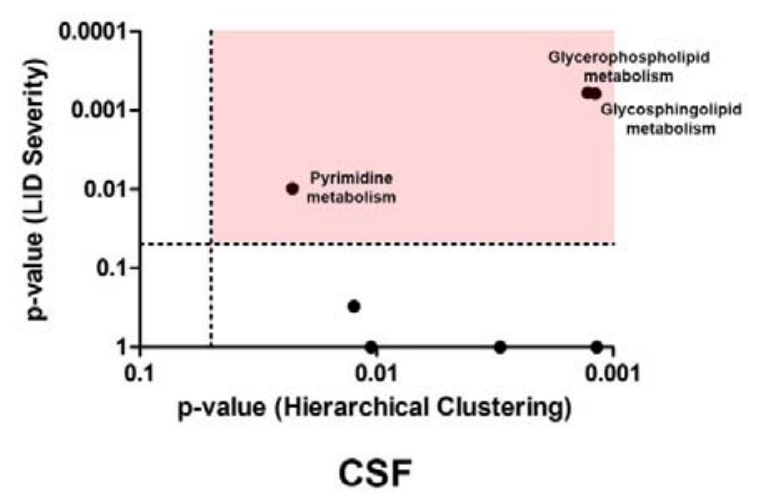

A: Plasma. B: CSF. The association of metabolic pathways with LID presence was analyzed by hierarchical clustering, and the association of metabolic pathways with LID severity was analyzed by linear regression. Each black dot represents a metabolic pathway. Metabolic pathways more associated with both LID presence and dyskinesia severity are shown in the pink quadrant. Dotted lines in both axes indicate $\mathrm{p}$-value $=0.05$. 